\title{
Step-by-step capping and strain state of GaN/AIN quantum dots studied by grazing-incidence diffraction anomalous fine structure
}

\author{
J. Coraux,,${ }^{1,2, *}$ M. G. Proietti, ${ }^{3}$ V. Favre-Nicolin,,${ }^{1,2}$ H. Renevier, ${ }^{1,2}$ and B. Daudin ${ }^{1}$ \\ ${ }^{1}$ Commissariat l'Energie Atomique, Département de Recherche Fondamentale sur la Matière Condensée, SP2M/NRS, 17 rue des martyrs, \\ 38054 Grenoble Cedex 9, France \\ ${ }^{2}$ Université Joseph Fourier, BP 53, 38041, Grenoble Cedex 9, France \\ ${ }^{3}$ Departamento de Física de la Materia Condensada, Instituto de Ciencia de Materiales de Aragón, CSIC-Universidad de Zaragoza, \\ c. Pedro Cerbuna 12, 50009 Zaragoza, Spain
}

(Received 5 October 2005; revised manuscript received 6 February 2006; published 24 May 2006)

\begin{abstract}
The investigation of small-size embedded nanostructures, by a combination of complementary anomalous diffraction techniques, is reported. GaN quantum dots (QD's), grown by molecular beam epitaxy in a modified Stranski-Krastanow mode, are studied in terms of strain and local environment, as a function of the AlN cap layer thickness, by means of grazing-incidence anomalous diffraction. That is, the x-ray photon energy is tuned across the $\mathrm{Ga}$ absorption $K$ edge which makes diffraction chemically selective. Measurement of $h k l$ scans, close to the $\operatorname{AlN}(30 \overline{3} 0)$ Bragg reflection, at several energies across the Ga $K$ edge, allows the extraction of the Ga partial structure factor, from which the in-plane strain of GaN QD's is deduced. From the fixed- $Q$ energydependent diffracted intensity spectra, measured for diffraction-selected isostrain regions corresponding to the average in-plane strain state of the QD's, quantitative information regarding the composition and out-of-plane strain has been obtained. We recover the in-plane and out-of-plane strains in the dots. The comparison to the biaxial elastic strain in a pseudomorphic layer indicates a tendency to an overstrained regime.
\end{abstract}

DOI: 10.1103/PhysRevB.73.205343 PACS number(s): 61.10.Nz, 61.10.Ht, 61.10.Eq, 61.46.-w

\section{INTRODUCTION}

Most of the interest in the improvement of the growth techniques for III-V semiconductor nanostructures originates from the fact that the quantum confinement of carriers leads to unique optoelectronic performances. The confinement in one dimension - that is, the growth of quantum wells (QW's) - has been brought under control for a variety of systems, leading to QW's based optoelectronic devices, such as nitride QW laser diodes. ${ }^{1}$ However, due to the large densities of defects in III-nitride materials, ${ }^{2}$ the emission efficiency of such devices is strongly altered by increasing the temperature. As an alternate way to overcome that difficulty, carriers may be confined in regions free of any defect, ${ }^{3}$ such as self-organized quantum dots (QD's). For reasonable optoelectronic efficiency, simultaneous control over the size, size distribution, nucleation sites, density, and structure of the QD's is required. In the InAs/GaAs system, roomtemperature ultraviolet lasers ${ }^{4}$ could be achieved once these requirements were satisfied. This article focuses on the GaN/AlN system, for which the overall control of the QD characteristics still remains a challenge. In the last few years, molecular beam epitaxy (MBE) has recently been of particular interest with the improvement of QD density control, using the Stranski-Krastanow (SK) growth mode ${ }^{5-7}$ and derivatives. $^{8-10}$ Size homogeneization of the QD's was achieved using vertical correlation through strain fields. ${ }^{11-13}$

Alternatively, new efforts are made to understand the effects of the QD capping by AlN, which strongly modifies the strain state in the QD's (Refs. 13 and 14) and therefore plays a decisive role in modification of the optical properties. Moreover, this topic raises a fundamental interest regarding the physics of strain accomodation between a QD and its capping.
Many complementary methods have been applied to quantitative strain characterization in nanostructures. ${ }^{15}$ This is true for grazing-incidence $\mathrm{x}$-ray diffraction (GIXRD), which can be made chemically sensitive when carried out as a function of the energy across the absorption edge of an element. This technique is known as anomalous diffraction ${ }^{16}$ and is suitable (a) to localize some element in reciprocal space, ${ }^{17}$ (b) to identify the local environment of an atom, ${ }^{18}$ and (c) to determine the strain and composition of an isostrain region selected by diffraction. ${ }^{19}$

In this article we present a comprehensive strain analysis of the capping of GaN QD's by AlN. More precisely, we study the in-plane and out-of-plane strain states and composition in the QD's as a function of the AlN capping thickness, by means of grazing-incidence anomalous x-ray diffraction at the $\mathrm{Ga} K$ edge $(10.367 \mathrm{keV})$, around the $(30 \overline{3} 0)$ reflection.

The MBE preparation of the set of samples to be analyzed is presented in Sec. II. The strain characterization technique, by grazing-incidence diffraction anomalous fine structure (GIDAFS), is detailed in Sec. III. The strain and composition information obtained using different aspects of GIDAFSnamely, multiwavelength anomalous diffraction (MAD), extended diffraction anomalous fine structure (EDAFS) oscillations, and diffraction anomalous line-shape analysis are given in Secs. IV-VI.

\section{SAMPLES}

The samples were grown in a MECA $2000 \mathrm{MBE}$ chamber, equipped with standard effusion cells providing the $\mathrm{Ga}$ and Al fluxes and a radiofrequency plasma cell providing the active nitrogen flux. The substrates were $2-\mu \mathrm{m}$-thick 


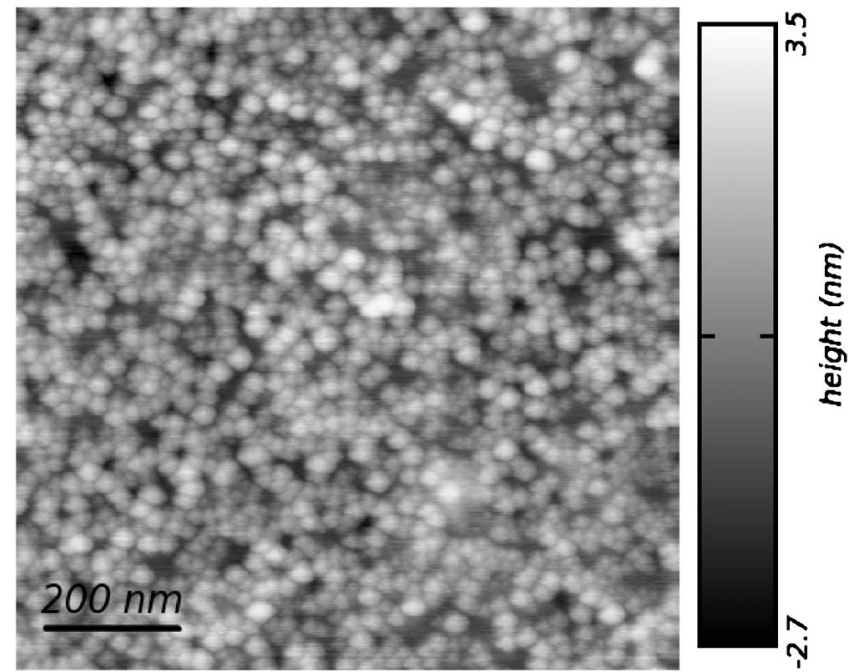

FIG. 1. 1- $\mu \mathrm{m}^{2} \mathrm{AFM}$ images of uncapped GaN QD's grown in the modified SK mode.

AlN(0001) layers deposited by metal-organic chemical vapor deposition on sapphire. ${ }^{20}$ The substrate temperature was fixed at $740{ }^{\circ} \mathrm{C}$. Prior to the growth of the QD's, 10-nmthick AlN buffers were grown. The QD growth was achieved in the modified SK growth mode, ${ }^{8,9}$ by depositing six $\mathrm{GaN}$ monolayers (ML) under Ga-rich conditions. This resulted in the formation of a Ga bilayer at the surface inhibiting the two- to three-dimensional (2D-3D) transition even above the usual $\sim 2 \mathrm{ML}$ critical thickness for the 2D-3D transition in the SK growth mode. ${ }^{5}$ The thermal evaporation under vacuum of the $\mathrm{Ga}$ bilayers. led to the transition of the $2 \mathrm{D}$ GaN layer into 3D QD's (Refs. 8 and 9) connected by a $\sim 2$-ML-thick wetting layer (WL). A set of five samples was grown, with increasing AlN capping: 0, 2, 5, 10, and 20 ML. Figure 1 shows a $1-\mu \mathrm{m}^{2}$ atomic force microscopy (AFM) image of free-standing dot samples. The height of the QD's was evaluated to $3.0 \pm 0.5 \mathrm{~nm}$ and their diameter to $15 \pm 1 \mathrm{~nm}$, leading to an aspect ratio of about 0.2 . The QD density was found as high as $1.3 \times 10^{11} \mathrm{~cm}^{-2}$; that is, QD's are almost adjacent. The roughness of the samples was measured to follow the evolution of the morphology as a function of the AlN deposit. Figure 2 shows the evolution of the root-mean-square (rms) roughness for $0,2,5$, and 10AlN ML deposited. The rms roughness remains roughly the same until a 5-ML deposit and drops for the 10-ML sample.

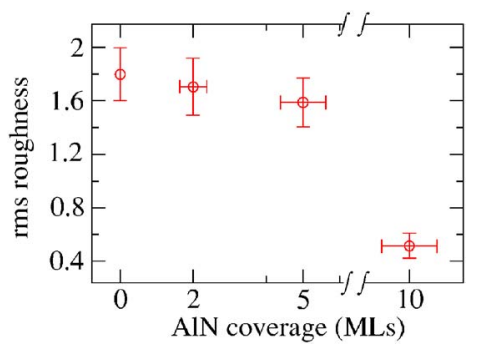

FIG. 2. (Color online) rms roughness evolution as a function of the AlN deposit amount.

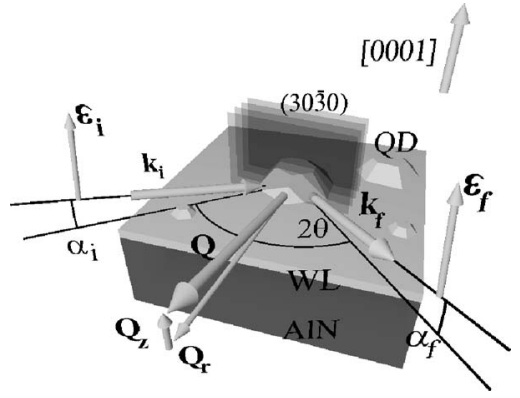

FIG. 3. Grazing-incidence geometry for a in-plane reflection $(30 \overline{3} 0)$. See text for details.

\section{GIDAFS MEASUREMENTS}

Grazing-incidence diffraction anomalous fine structure at the Ga $K$ edge $(10.367 \mathrm{keV})$ was performed at the French Collaborative Research Group beamline BM2 at the European Synchrotron Radiation Facility (ESRF) by using the eight-circle diffractometer equipment. We measured the diffuse scattering intensity, in grazing incidence and exit, close to the in-plane $(30 \overline{3} 0)$ Bragg reflection of the AlN substrate (radial scans), at energies close to the Ga $K$ edge. Figure 3 sketches the experimental setup. The samples were mounted in the vertical plane; i.e., the polarization vector $\widehat{\varepsilon_{i}}$ of the incident photon beam was perpendicular to the sample surface (0001). The incidence angle was $\alpha_{i}=0.17^{\circ}$, lower than the bulk AlN critical angle $\alpha_{c}=0.21^{\circ}$ (at $10.32 \mathrm{keV}$ ) for which the total reflection regime takes place. Such conditions were used to enhance the weak contribution of the encapsulated dot layer with respect to that of the substrate. The diffraction geometry was chosen in such a way as to keep the scattering vector in the vertical plane. The diffraction point detector was a scintillator, and the slits were opened so as to measure the integrated intensity over the grazing exit angle $\alpha_{f}$. A photodiode measuring the fluorescence yield of an invacuum $4-\mu \mathrm{m}$ Ti foil was used to monitor the incoming x-ray beam. Two kinds of scans were performed: (a) $h$ scans (radial) in the range 2.9-3.05 for 12 energies, from 10.272 to $10472 \mathrm{keV}$-i.e., close to the $\mathrm{Ga} K$ edge-and (b) energy scans at fixed scattering vector $(\mathbf{Q})$ corresponding to the maximum of the QD contribution to the diffuse scattering (i.e., at the maximum of the partial structure factor $F_{A=\mathrm{Ga}}$ profile extracted from the multiwavelength $h$ scans; see the following section and Ref. 19). The energy scans were recorded in a large energy interval, typically $1 \mathrm{keV}$, with an energy step from 1 to $2 \mathrm{eV}$, to allow a quantitative analysis of both the edge and extended oscillations.

\section{MAD ANALYSIS}

The solid lines in Figs. 4(a), 4(c), and 4(e) show the square-root intensities along the $[10 \overline{1} 0]$ direction ( $h$ scan) close to the $(30 \overline{3} 0)$ reflection as a function of the AlN coverage. These $h$ scans are related to both the in-plane strain state and size. With no AlN coverage, one observes a diffusescattering peak ascribed to QD's slightly strained by the AlN 


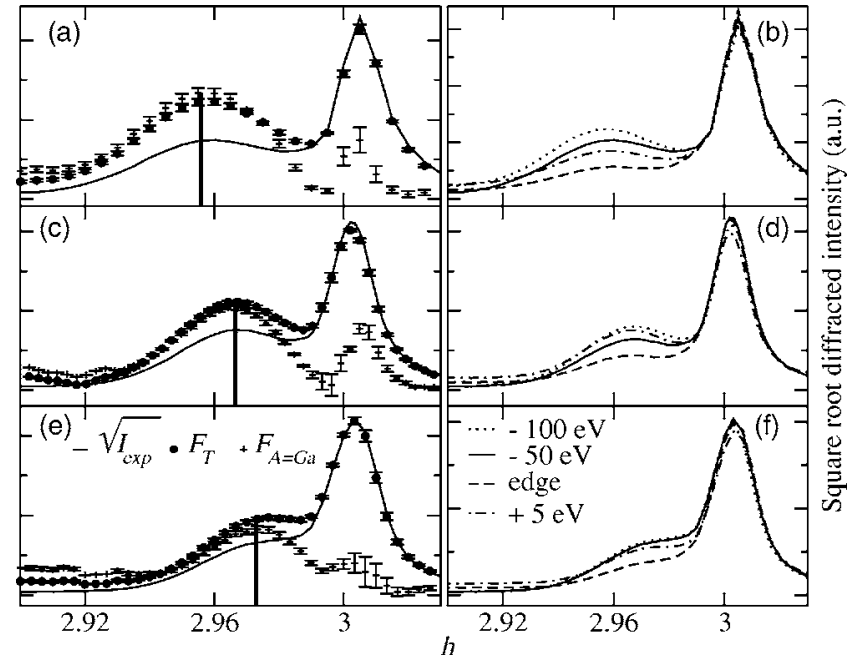

FIG. 4. (a), (c), (e) $\sqrt{I_{\text {exp }}}$ measured at $10.317 \mathrm{keV}(50 \mathrm{eV}$ below the Ga $K$ edge), $\left\|F_{\mathrm{Ga}}\right\|$ and $\left\|F_{T}\right\|$ extracted for a 0 -ML (a), a 5-ML (c), and a 10-ML (e) AlN coverage. (b), (d), (f) experimental square-root intensities $\sqrt{I_{\text {exp }}}$ measured below ( -100 and $\left.-50 \mathrm{eV}\right)$, at (edge), and above $(+5 \mathrm{eV})$ the $\mathrm{Ga} K$ edge for a $0-\mathrm{ML}$ (b), a 5-ML (d), and a 10-ML (f) AlN coverage.

buffer and substrate. As the AlN coverage increases [from (a) free-standing QD's to (e) 10-ML AIN coverage], this peak is progressively shifted towards higher $h$ values and gets mixed with the AlN buffer peak. Further analysis was made possible by distinguishing the GaN and AlN contributions, using MAD measurements. ${ }^{16,17,19}$ Figures 4(b), 4(d), and 4(f) show some of the square-root diffracted intensities measured for increasing AlN coverage, across the Ga $K$ edge, taking advantage of the Ga anomalous effect to localize the Ga contribution along [10 $\overline{1} 0]$. The Ga scattering factor can be written as $f_{\mathrm{Ga}}=f_{\mathrm{Ga}}^{0}+f_{\mathrm{Ga}}^{\prime}+i f_{\mathrm{Ga}}^{\prime \prime}$, where $f_{\mathrm{Ga}}^{\prime}$ and $f_{\mathrm{Ga}}^{\prime \prime}$ are the Ga real and imaginary anomalous (resonant) scattering corrections and $f_{\mathrm{Ga}}^{0}$ is the $\mathrm{Ga}$ Thomson scattering factor. From MAD measurements, the Ga partial structure factor $F_{\mathrm{Ga}}$ of phase $\varphi_{\mathrm{Ga}}$, which includes the Thomson scattering of all anomalous atoms $(\mathrm{Ga})$, can be retrieved. The retrieval shall be run in the framework of the distorted-wave Born approximation, taking into account scattering paths involving the reflection from the layer supporting the dots. ${ }^{21,22}$ We recorded the diffracted intensity integrated over the exit angle $\alpha_{f}$, between 0 and $2 \alpha_{c}$, and therefore collected all the scattering paths. Discarding the energy dependence of the reflection coefficients at the $\mathrm{Ga} K$ edge, as a consequence of the small $\mathrm{Ga}$ amount (six equivalent monolayers), the recorded intensity corrected for fluorescence, $I_{\text {exp }}$, is proportional to the total square structure factor $\|F\|^{2}$ :

$$
\begin{aligned}
I_{\text {exp }}(E) \propto & \|F\|^{2} \propto\left\|F_{T}\right\|^{2}\left\{\left[\cos \left(\varphi_{T}-\varphi_{\mathrm{Ga}}\right)+\beta f_{\mathrm{Ga}}^{\prime}\right]^{2}\right. \\
& \left.+\left[\sin \left(\varphi_{T}-\varphi_{\mathrm{Ga}}\right)+\beta f_{\mathrm{Ga}}^{\prime \prime}\right]^{2}\right\},
\end{aligned}
$$

where $\beta=\left\|F_{\mathrm{Ga}}\right\| /\left(f_{\mathrm{Ga}}^{0}\left\|F_{T}\right\|\right)$. Figure 5 shows the total and partial structure factor relations in the complex plane. The partial structure factor $F_{T}$ of phase $\varphi_{T}$ that includes the overall contribution of nonanomalous atoms and the Thomson scattering of all anomalous atoms, $F_{\mathrm{Ga}}$, as well as $\varphi_{T}-\varphi_{\mathrm{Ga}}$, can

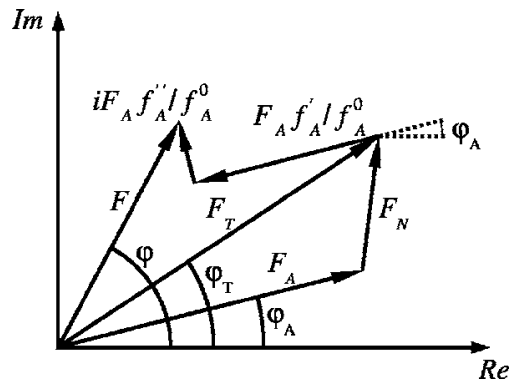

FIG. 5. Schematic representation in the complex plane of the structure factor $F$ as a function of $F_{T}, F_{A=\mathrm{Ga}}$, and $\varphi_{T}-\varphi_{A=\mathrm{Ga}}$ (see text). $F_{N}$ represents the partial structure factor of nonresonant atoms $(\mathrm{Al}, \mathrm{N})$.

be extracted for all $h$ values, without any structural model by fitting Eq. (1) to the experimental data with the NANOMAD algorithm. $^{23}$

As shown in Figs. 4(a), 4(c), and 4(e), $F_{\mathrm{Ga}}$ and $F_{T}$ were extracted. The $h=h_{\mathrm{Ga}}$ position of the diffuse $F_{\mathrm{Ga}}$ peak maximum is inversely proportional to the in-plane average lattice parameter $a_{\mathrm{GaN}}$, since the distance between $\mathrm{GaN}$ (30 $\overline{3} 0)$ planes is $d_{30 \overline{3} 0}=(\sqrt{3} / 2) a_{\mathrm{GaN}} / 3=(\sqrt{3} / 2) a_{\mathrm{AlN}} / h_{\mathrm{Ga}}$ with $a_{A l N} \simeq 3.112 \AA$, for the AlN substrate peak used as a reference. Figure 6 shows the evolution of the in-plane lattice parameter as a function of the AIN cap thickness. The uncapped QD's are partially in-plane relaxed, with an average strain relative to bulk GaN, $\varepsilon_{x x, G I X R D}=\left(a_{\mathrm{GaN}, \text { GIXRD }}\right.$ $\left.-a_{\mathrm{GaN}, \text { bulk }}\right) / a_{\mathrm{GaN}, \text { bulk }} \sim-1 \%$, with $a_{\mathrm{GaN}, \text { bulk }}=3.189 \AA$. The QD's are then progressively in-plane compressed by the AlN capping, but remain slightly relaxed, $\varepsilon_{x x, G I X R D} \sim-1.6 \%$, compared to pseudomorphic $\mathrm{GaN}\left(\varepsilon_{x x} \sim-2.4 \%\right)$.

\section{EDAFS ANALYSIS}

Out-of-plane information can be achieved by a quantitative analysis of the grazing-incidence diffraction anomalous fine structure oscillations in the extended region above the edge (EDAFS). Figure 7(a) shows for free-standing QD's the oscillatory contribution $\left(\chi_{D A F S}\right)$ to the DAFS spectrum, extracted and normalized to the smooth atomic background $\left(I_{0}\right)$ :

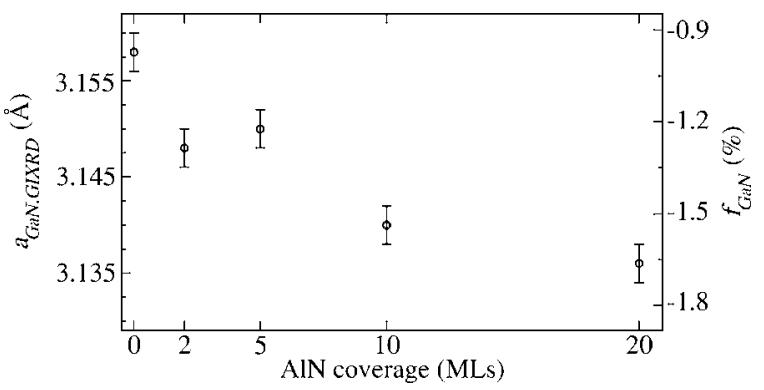

FIG. 6. In-plane lattice parameter $a_{\mathrm{GaN}, G I X R D}$ and strain (relative to bulk $\mathrm{GaN}$ ) in $\mathrm{GaN}$ deduced from the position of the $F_{\mathrm{Ga}}$ maximum. Bulk GaN gives $\varepsilon_{x x, G I X R D}=0 \%$ with $a_{\mathrm{GaN}, \text { bulk }}=3.189 \AA$ 品 while bulk AlN gives $\varepsilon_{x x, G I X R D}=-2.4 \%$ with $a_{\mathrm{AlN}}=3.112 \AA$. 


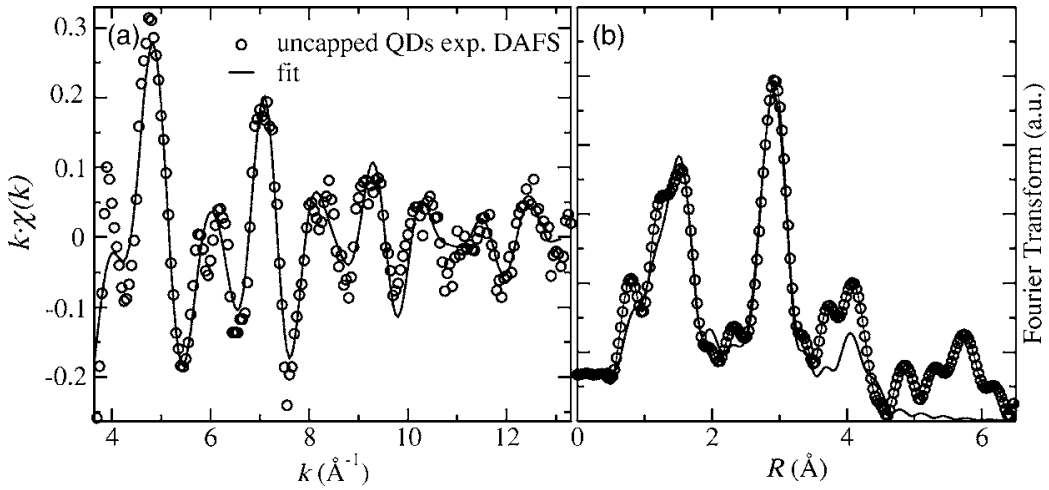

FIG. 7. (a) Experimental EDAFS for the freestanding QD sample, compared with the best-fit result and (b) $R$-space experimental curve for free-standing QD's compared with the best fit.

$$
\chi_{D A F S}=\frac{I_{\exp }-I_{0}}{I_{0}} .
$$

$\chi_{D A F S}$ can be written as $\chi_{D A F S}=\frac{1}{S_{D}} \chi_{Q}$ where $S_{D}$ is a normalization factor that depends on crystallography and is calculated from the parameters $\Delta \varphi=\varphi_{T}-\varphi_{A}$ and $\beta$ [see Eq. (1)] and $\chi_{Q}$ is in the first-order approximation of the diffracted anomalous fine structure: ${ }^{24}$

$$
\chi_{\mathbf{Q}}(k)=\cos \left(\varphi_{0}-\varphi_{A}\right) \sum_{j=1}^{N_{A}} w_{j}^{\prime} \chi_{j}^{\prime}+\sin \left(\varphi_{0}-\varphi_{A}\right) \sum_{j=1}^{N_{A}} w_{j}^{\prime \prime} \chi_{j}^{\prime \prime},
$$

where $\varphi_{0}$ is the phase of the smooth structure factor (without oscillations), the $j$ label runs over the different anomalous sites $A$ (the $\mathrm{Ga}$ sites, $A=\mathrm{Ga})$ and $w_{j}^{\prime}=\frac{\left\|F_{j}\right\| \cos \left(\varphi_{0}-\varphi_{j}\right)}{\left\|F_{A}\right\| \cos \left(\varphi_{0}-\varphi_{A}\right)}$ and $w_{j}^{\prime \prime}$ $=\frac{\left\|F_{j}\right\| \sin \left(\varphi_{0}-\varphi_{j}\right)}{\left\|F_{A}\right\| \sin \left(\varphi_{0}-\varphi_{A}\right)}$ are crystallographic weights. The term $\chi_{j}^{\prime}\left(\chi_{j}^{\prime \prime}\right)$ in Eq. (2) is the oscillatory part of the resonant atomic scattering factor $f_{j}^{\prime}\left(f_{j}^{\prime \prime}\right)$; it is related to the local atomic environment of the resonant atom. $\chi_{j}^{\prime \prime}$ is formally identical to the extended $\mathrm{x}$-ray absorption fine structure (EXAFS) oscillations of atom $j$. In the present case-i.e., one statistically equivalent site $-\chi_{Q}$ can be rewritten as a function of the virtual photoelectron wave-vector modulus $k$ in a form that is similar to the well-known EXAFS formula: ${ }^{24}$

$$
\begin{aligned}
& \chi_{\mathbf{Q}}(k) \\
& \quad=\sum_{\gamma} A_{\gamma}(k) \sin \left[2 k\langle R\rangle_{\gamma}+\varphi_{\gamma}(k)+2 \delta_{c}(k)+\varphi_{0}-\varphi_{A}-\frac{\pi}{2}\right],
\end{aligned}
$$

where $\gamma$ runs over all possible virtual photoelectron scattering paths, $\langle R\rangle_{\gamma}$ is the effective length of path $\gamma$, and $\varphi_{\gamma}(k)$ $+2 \delta_{c}(k)$ is the net scattering photoelectron phase shift.

The analysis can be performed according to the standard criteria and available codes for EXAFS, provided that crystallographic phases and amplitude correction factors are taken into account (for more details see Refs. 24 and 25). The EDAFS analysis has been carried out by using the FEFF8 code ${ }^{26}$ to generate theoretical phases and amplitudes, taking into account beam polarization, for a $6-\AA$-radius GaN cluster. In order to address the possible presence of $\mathrm{Al}$ atoms in the QD's or at the substrate and capping interface, Ga-Al and
Ga-N-Al scattering paths were considered by calculating an AlN cluster with the Ga central atom as absorber. The ARTEMIS interface to the IFEFFIT package ${ }^{27}$ was used to fit theoretical computations to the experimental data.

The EDAFS spectra were Fourier transformed in the $k$ range $3-10 \AA^{-1}$, and the fit was performed in $R$ space (real space), using four next-neighboring shells (I-IV). As an example, we show the best-fit curves for free-standing QD's in Figs. 7(a) and 7(b), compared to the experimental raw data. Six single scattering (SS) paths and four multiple-scattering (MS) paths were found to be relevant in this range (see Fig. 8).

(i) $(\mathrm{Ga}-\mathrm{N})_{\|}$, in-plane, I-shell path, corresponding to the three $\mathrm{Ga}-\mathrm{N}$ bonds of the tetrahedron that are nearly in plane.

(ii) $(\mathrm{Ga}-\mathrm{N})_{\perp}$, out-of-plane, I-shell path, corresponding to the fourth $\mathrm{Ga}-\mathrm{N}$ bond of the tetrahedron, lying along the $c$ axis.

(iii) Ga-Ga, II-shell, out-of-plane path, corresponding to six $\mathrm{Ga}$ atoms at a distance that is a combination of $a$ and $c$, $\left\{\frac{1}{3} a_{\text {GIXRD }}^{2}+\frac{1}{4} c^{2}\right\}^{1 / 2}$, where $a_{\text {GIXRD }}$ is the in-plane lattice pa-
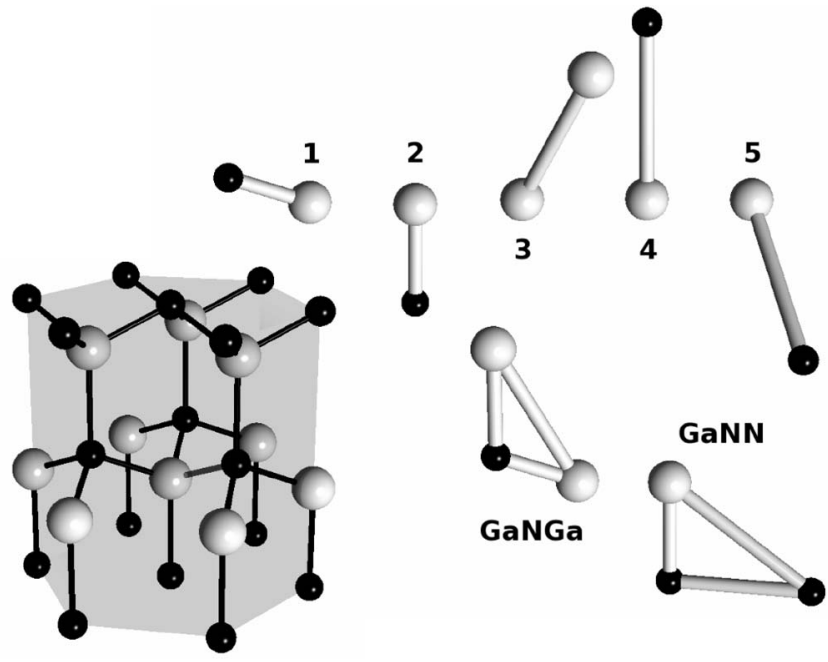

GaNN

GaNGa

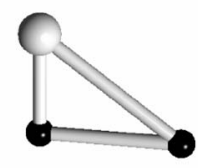

FIG. 8. Scheme of GaN wurzite structure; the most relevant virtual phoelectron scattering paths used for the EDAFS simulation are represented: (1) in-plane $I$-shell $(\mathrm{Ga}-\mathrm{N})_{\|},(2)$ out-of-plane $I$-shell $(\mathrm{Ga}-\mathrm{N})_{\perp}$, (3) out-of-plane II-shell $(\mathrm{Ga}-\mathrm{Ga})_{\perp}$, (4) III shell Ga-N along $c$, (5) nearly in-plane IV-shell Ga-N, MS Ga-N-N, and $\mathrm{Ga}-\mathrm{N}-\mathrm{Ga}$. $\mathrm{Ga}$ atoms are represented by white spheres, $\mathrm{N}$ by black ones. 
TABLE I. EDAFS best-fit values for interatomic distances $(R)$, Debye-Waller factors $(\sigma)$, and Al concentration $\left(x_{\mathrm{Al}}\right)$ obtained by IFEFIT minimization using theoretical fitting standards provided by the FEFF8 code. The amplitude and phase correction factors have been obtained by crystallographic analysis of the DAFS line shape. The $a_{\mathrm{GaN}, \text { GIXRD }}$ value is kept fixed to the value determined by grazing incidence and exit diffraction (diff.).

\begin{tabular}{lcccccc}
\hline \hline & Bulk & GaN/AlN & 0 MLs & 2 MLs & 5 MLs & 10 MLs \\
\hline $\mathrm{R} 1(\mathrm{Ga}-\mathrm{N})(\AA)$ & - & - & 1.93 & 1.94 & 1.94 & 194 \\
$\sigma_{1}^{2}\left(\AA^{2}\right)$ & - & - & $2 \times 10^{-3}$ & $4 \times 10^{-3}$ & $4 \times 10^{-3}$ & $1 \times 10^{-3}$ \\
$\mathrm{R} 2(\mathrm{Ga}-\mathrm{Ga})_{\|}=a_{\mathrm{GaN}}(\AA)$ & 3.188 & 3.11 & $3.156($ diff. $)$ & $3.147($ diff. $)$ & 3.149 (diff. $)$ & 3.14 (diff.) \\
$\sigma_{1}^{2}(\AA)^{2}$ & - & - & $6 \times 10^{-3}$ & $8 \times 10^{-3}$ & $4 \times 10^{-3}$ & $7 \times 10^{-3}$ \\
$\mathrm{R} 2(\mathrm{Ga}-\mathrm{Ga})(\AA)$ & 3.18 & - & 3.19 & 3.18 & 3.18 & 3.19 \\
$c_{\mathrm{GaN}}(\AA)$ & 5.186 & 5.26 & $5.25 \pm 0.02$ & $5.23 \pm 0.03$ & $5.22 \pm 0.02$ & $5.25 \pm 0.04$ \\
$c_{\mathrm{GaN}} / a_{\mathrm{GaN}}$ & 1.626 & 1.69 & 1.66 & 1.66 & 1.66 & 1.67 \\
$x_{\mathrm{Al}}$ & - & - & $0.1 \pm 0.1$ & $0.0 \pm 0.1$ & $0.1 \pm 0.1$ & $0.05 \pm 0.1$ \\
\hline \hline
\end{tabular}

rameter obtained with the grazing-incidence (and exit) x-ray diffraction experiment (see Sec. IV).

(iv) Ga-N, III-shell path, corresponding to one $\mathrm{N}$ atom along the $c$ direction.

(v) Ga-N, IV-shell path, corresponding to six $\mathrm{N}$ nearly in-plane atoms.

(vi) MS paths consisting of triangular paths Ga-N-N and Ga-N-Ga.

The "in-plane" statement refers to the surface or growth plane, and all the scattering paths, except the first one, were expressed in terms of $a$ and $c$ cell parameters, as requested by the hexagonal cell symmetry.

We performed the fit by fixing the $a$ parameter (in plane) to the values found by diffraction, $a_{\mathrm{GaN}, \text { GIXRD }}$, letting the $c$ parameter vary according to the hexagonal symmetry. The Ga-N first-shell distances were let free to vary independently of $a$ and $c$ since, as is well known, Vegard's law is far from being valid for semiconductor alloys, in which the bondbending mechanism is dominant compared to bond stretching. ${ }^{28}$ The presence of $\mathrm{Al}$ is taken into account by adding the correspondent scattering paths in which $\mathrm{Al}$ substitutes for Ga as next nearest neighbor (NNN) and multiplying the amplitude by a factor $x_{\mathrm{Al}}$ for $\mathrm{Al}$ and $\left(1-x_{\mathrm{Al}}\right)$ for $\mathrm{Ga}$. The best-fit parameters are shown for the whole set of samples in Table I, where we also report, as a reference, the bulk and pseudomorphic values for GaN. ${ }^{29}$ The Ga-Al distance was also let free to vary and the values found were close to the Al-Al NNN distance. Since the Al content is found to be zero within the statistical errors, the Al-Al NNN distance was not reported in the table. Starting from the fit results, the in-plane and out-of-plane strains were calculated, with respect to relaxed (bulk) $\mathrm{GaN}$, as $\varepsilon_{x x, \text { GIXRD }}=\left(a_{\mathrm{GaN}, \text { GIXRD }}-a_{\mathrm{GaN}, \text { bulk }}\right) / a_{\mathrm{GaN}, \text { bulk }}$ and $\varepsilon_{z z, \text { GIDAFS }}$ $=\left(c_{\mathrm{GaN}, \text { GIDAFS }}-c_{\mathrm{GaN}, \text { bulk }}\right) / c_{\mathrm{GaN}, \text { bulk }}$. $\varepsilon_{x x, G I X R D}$ vs $\varepsilon_{z z, G I X R D}$ for the different samples studied is sketched in Fig. 9. These values are compared to the biaxial elastic behavior for pseudomorphic GaN on AlN (straight line), which corresponds to $\varepsilon_{x x}=-2 \varepsilon_{z z} c_{13} / c_{33}$, with the elastic coefficients $c_{13}$ and $c_{33}$ values from Ref. 30 .

We observe the following general findings.

(i) The Ga-N first-shell in-plane and out-of-plane distances are seen to be very close to each other, within the fit errors $(0.01 \AA)$, in agreement with previous studies. ${ }^{31}$

(ii) As shown in Table I, the Al content remains very small, showing that no intermixing takes place in the QD's as expected for the $\mathrm{Al} / \mathrm{Ga}$ species. ${ }^{32}$

(iii) The $c_{\mathrm{GaN}, \text { GIDAFS }}$ values range from 5.22 to $5.25 \AA$, which is quite large compared to the values foreseen by the elastic regime of a pseudomorphic GaN layer, as apparent in Fig. 9 where the experimental points fall above the elasticity curve.

As expected, the uncapped QD's do not follow a biaxial strain behavior, because of the presence of a free surface. However, capping by a thin layer (2-5 ML) of AlN should favor evolution towards the biaxial case for at least two reasons: (a) first of all, capping results in a size decrease of the dots-i.e., an aspect ratio reduction associated with a relative increase of the biaxial component of the $\operatorname{strain}^{33}$ - (b) furthermore, we speculate that the possible wetting of the QD's by AlN, which will be discussed in Sec. VI, strongly tends to reduce the relaxation through the free surface, which also results in a relative increase of the biaxial component of the strain. Besides these considerations, it is worth noting that the plastic relaxation process of AlN deposited on GaN, which is characterized by a very small critical thickness, ${ }^{34}$ is still unclear and may also determine to some extent the strain state of the AIN/GaN QD interacting system.

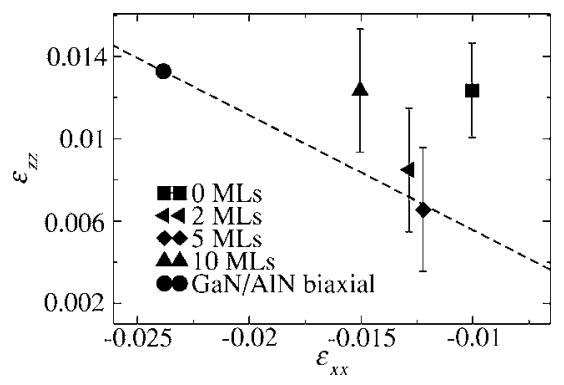

FIG. 9. GaN QD strain $\varepsilon_{x x}=\left(a_{\mathrm{GaN}, \text { GIXRD }}-a_{\mathrm{GaN}, \text { bulk }}\right) / a_{\mathrm{GaN}, \text { bulk }} \mathrm{vs}$ $\varepsilon_{z z}=\left(c_{\mathrm{GaN}, \text { GIDAFS }}-c_{\mathrm{GaN}, \text { bulk }}\right) / c_{\mathrm{GaN}, \text { bulk }}$ values for all the samples studied compared with elastic biaxial strain of a pseudomorphic GaN thin film. 


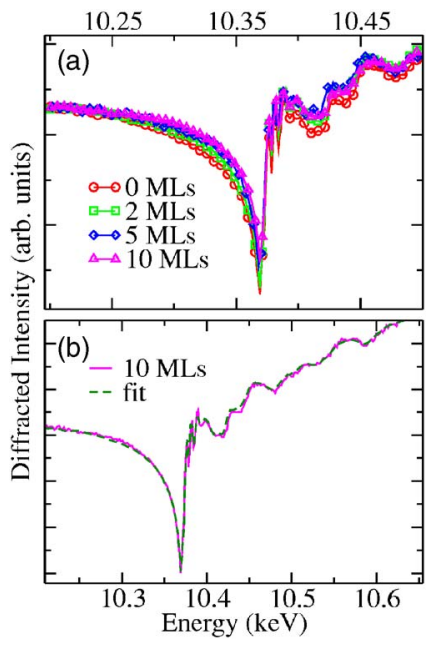

FIG. 10. (Color online) (a) GIDAFS spectra for 0-, 2-, 5-, and 10-ML AlN capping, measured at maximum of $F_{A}$ and (b) crystallographic best fit for the $10 \mathrm{ML}$ AlN sample. Open circle: experiment. Solid line: simulation performed with experimental $f_{\mathrm{Ga}}^{\prime}$ and $f_{\mathrm{Ga}}^{\prime \prime}$ of a GaN thin film.

\section{EDGE ANALYSIS}

The diffraction anomalous spectra, close to the Ga $K$ edge, can give the $\mathrm{Al}$ and $\mathrm{Ga}$ relative compositions inside the GaN/AlN in-plane isostrain region selected with grazing incidence and exit diffraction; this region includes the $\mathrm{GaN}$ QD's and the AIN on top. Previous studies clearly indicate that no atomic intermixing occurs at the GaN/AlN interfaces, with neither GaN/AlN QW's nor GaN/AIN QD's. ${ }^{32,33}$ This is confirmed by the grazing-incidence EDAFS results reported in Sec. V, which clearly show no significant $\mathrm{Al} / \mathrm{Ga}$ mixing.

On the other side, analysis of the DAFS edge shape can give information about the capping mechanism of the QD's. Indeed, as a first approximation, the diffracted intensity is proportional to the square modulus of the in-plane isostrain region structure factor. We calculate this structure factor for an $\mathrm{Al}_{x} \mathrm{Ga}_{1-x} \mathrm{~N}$ wurtzite structure to take into account the $\mathrm{Al}$ atoms belonging to the same isostrain region as the $\mathrm{Ga}$ atoms at the QD top. The Al concentration obtained by refining the $x$ value gives the $\mathrm{Al}$ atom fraction seen by diffraction, contributing at the chosen $Q$ value and determining the edge line shape. Taking into account that EDAFS analysis shows that no intermixing takes place, we can state that we are probing the AlN capping. Figure 10(a) shows the GIDAFS spectra for the $0,2,5$, and 10 AlN ML cap thicknesses, measured at the maximum of the partial structure factor $F_{A=\mathrm{Ga}}$ (see Sec. IV). The data were normalized so that the intensity at $10.2 \mathrm{keV}$ is the same for all spectra. Equation (1) was fitted to each GIDAFS spectrum, using the anomalous scattering factors $f_{\mathrm{Ga}}^{\prime}$ and $f_{\mathrm{Ga}}^{\prime \prime}$ of a GaN layer. A scale factor, the detector efficiency as a function of the energy and the Al occupation factor $(x)$ inside the in-plane isostrain region, was refined. As an example, Fig. 10(b) shows the best fit for the 10-ML sample obtained with $x=0.39 \pm 0.01$. It should be noted that the occupation factor is determined by the ratio $\beta$

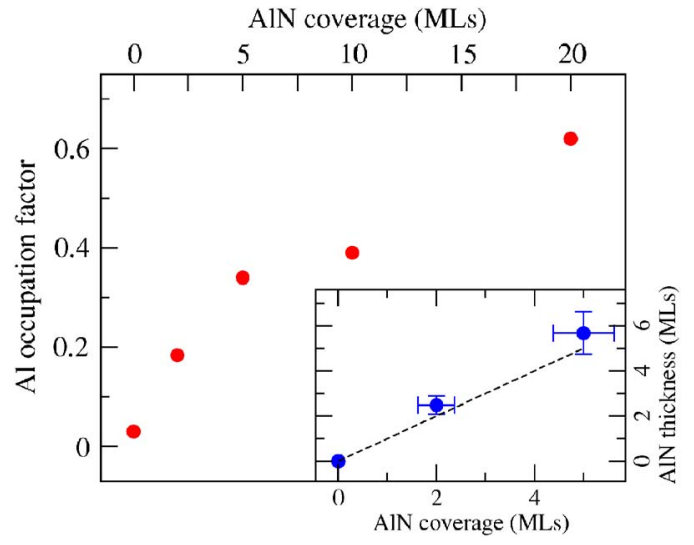

FIG. 11. (Color online) Al-atoms occupation factor $x$ of the $\mathrm{Al}_{x} \mathrm{Ga}_{1-x} \mathrm{~N}$ isostrain region selected by diffraction (maximum of $\left.F_{\mathrm{Ga}}\right)$, as a function of AlN coverage. Inset: evolution of the AlN thickness on top of the dots plotted as a function of the nominal AlN deposit (see text). The dashed straight line of slope 1 corresponds to an AlN quantity on dots equal to the nominal AIN deposit.

$=\frac{\left\|F_{A}\right\|}{f_{\mathrm{Ga}}^{0}\left\|F_{T}\right\|}$-i.e., the curvature and depth of the cusp before and at the edge. The fit quality is the same for all samples. Figure 11 shows the $\mathrm{Al}$ concentration $(x)$ as a function of the AlN cap thickness (nominal value). For free-standing dots, the AlN contribution is near to $0, x=0.03 \pm 0.01 \%$, as an indication that AlN from the buffer underneath the dots has no contribution to the isostrain region. Up to $5 \mathrm{ML}$ the $\mathrm{AlN}$ contribution increases linearly, stabilizes above $5 \mathrm{ML}$, and at $10 \mathrm{ML}$ the contribution is almost the same as for the 5-ML sample. Provided that AIN on top of the QD's is pseudomorphic to $\mathrm{GaN}$ for low coverages, ${ }^{13}$ the evolution of the $\mathrm{Al}$ concentration $(x)$ in the isostrain region up to $5 \mathrm{ML}$ indicates a linear increase of the amount of AlN on top of the GaN QD's. The inset of Fig. 11 shows the average thickness of the AlN deposit on top of the QD's as a function of the nominal cap thickness (up to $5 \mathrm{ML}$ ). This value was estimated using the mean diameter and mean height of the uncovered GaN QD's. It is remarkable that up to about $5 \mathrm{ML}$, the calculated AlN layer thickness is very close to that of the nominal AlN deposit. The dashed straight line of slope 1 corresponds to an AlN quantity on dots equal to the nominal AlN deposit. This result indicates a uniform growth of AlN on the sample surface, as also indicated by the AFM observations showing no noticeable surface morphology change below 5 ML (see Sec. II). Such AlN covering has already been observed, at least for very small deposits of AlN. ${ }^{33}$ The wetting of GaN QD's by AlN is supposedly driven by an Al-N bonding that is more stable than the Ga-N one, which leads to a vertical exchange between $\mathrm{Ga}$ and $\mathrm{Al}$. The further evolution of the $\mathrm{Al}$ concentration above $5 \mathrm{ML}$ (see Fig. 11) points out a change in the AlN growth process, leading to AlN with an in-plane strain state different from that in the QD's. This change may correspond either to plastic relaxation in AIN or to selective AlN growth in between of the QD's where the stress differs from that on top of the QD's or to another still unknown process. Whatever the process, it should lead to the flattening of the surface as shown by the AFM images for high AlN deposits. 


\section{CONCLUSION}

We have presented results on the structural properties of GaN QD's by combining different aspects of x-ray diffraction: quasi surface sensitivity due to grazing incidence, quantitative analysis of anomalous effects according to MAD principles, line-shape fit of DAFS and EDAFS oscillations fit.

All these aspects are strongly complementary. We determine in-plane and out-of-plane lattice parameters and investigate the effect of the capping layer by monitoring its effect on the QD strain. In addition, the $\mathrm{Al}$ fraction seen by the anomalous diffraction as a function of the capping layer thickness (obtained by GIDAFS line-shape analysis at the Ga $K$ edge) indicates a wetting of the QD's, followed by a noticeable change in the capping process which may be related either to plastic relaxation in AIN, to spatially selective AlN growth, or to a still unknown process. Let us point out that the $\mathrm{Al}$ fraction obtained in this way does not represent the $\mathrm{Al}$ content inside the dots, but the AlN contribution to the diffuse scattering at the same $\mathbf{Q}$ value as the GaN QD contribution-i.e., AlN mostly located on top of the QD's. The Al content of the dots can be found by analysis of the
EDAFS oscillations which provide the microscopic local environment of the Ga resonant atom. Our analysis shows that no $\mathrm{Ga} / \mathrm{Al}$ intermixing takes place, as expected for these two group-III-N elements. We recovered the in-plane and perpendicular strains $\varepsilon_{x x}$ and $\varepsilon_{z z}$ in the dots and compare them to the biaxial elastic strain of a pseudomorphic layer. We find a tendency to an overstrained regime that suggests a more complex mechanism of strain accommodation which deserves further investigations.

\section{ACKNOWLEDGMENTS}

We are very grateful to N. Boudet, S. Arnaud, B. Caillot, and J.F. Bérar for help in setting up the GIDAFS experiment at beamline BM2 at the ESRF. We are very grateful to P. Wolfers who gave us the DPU code used for data analysis. The authors would like to thank C. Priester for her critical reading of the manuscript. M.G.P. acknowledges the support of the Spanish Ministry of Education and Science in the frame of "Programa de estancias de profesores españoles en centros de investigación extranjeros" (Project No. PR20050231).
*Electronic address: Johann.Coraux@cea.fr

${ }^{1}$ F. A. Ponce and D. P. Bour, Nature (London) 386, 351 (1997).

${ }^{2}$ X. H. Wu, P. Fini, E. J. Tarsa, B. Heying, S. Keller, U. K. Mishra, S. P. DenBaars, and J. S. Speck, J. Cryst. Growth 189/190, 231 (1998).

${ }^{3}$ Y. Arakawa and H. Sakaki, Appl. Phys. Lett. 40, 939 (1982).

${ }^{4}$ N. Ledentsov, D. Bimberg, Z. I. Alferov, V. M. Ustinov, and J. A. Lott, Physica E (Amsterdam) 13, 871 (2002).

${ }^{5}$ B. Daudin, F. Widmann, G. Feuillet, Y. Samson, M. Arlery, and J. L. Rouvière, Phys. Rev. B 56, R7069 (1997).

${ }^{6}$ C. Adelmann, B. Daudin, R. A. Olivier, G. A. D. Briggs, and R. E. Rudd, Phys. Rev. B 70, 125427 (2004).

${ }^{7}$ J. Brown, F. Wu, P. M. Petroff, and J. S. Speck, Appl. Phys. Lett. 84, 690 (2004).

${ }^{8}$ N. Gogneau, D. Jalabert, E. Monroy, T. Shibata, M. Tanaka, and B. Daudin, J. Appl. Phys. 94, 2254 (2003).

${ }^{9}$ C. Adelmann, N. Gogneau, E. Saragiannidou, J.-L. Rouviére, and B. Daudin, Appl. Phys. Lett. 81, 3064 (2002).

${ }^{10}$ B. Damilano, N. Grandjean, S. Dalmasso, and J. Massies, Appl. Phys. Lett. 75, 3751 (1999).

${ }^{11}$ J. Tersoff, C. Teichert, and M. G. Lagally, Phys. Rev. Lett. 76, 1675 (1996).

${ }^{12}$ V. Chamard, T. H. Metzger, M. Sztucki, V. Holy, M. Tolan, E. Bellet-Amalric, C. Adelmann, B. Daudin, and H. Mariette, Europhys. Lett. 63, 268 (2003).

${ }^{13}$ J. Coraux, H. Renevier, V. Favre-Nicolin, G. Renaud, and B. Daudin, Appl. Phys. Lett. 88, 153215 (2006).

${ }^{14}$ B. Daudin, J.-L. Rouvière, D. Jalabert, J. Coraux, H. Renevier, C. Mann-Ho, C. Kwun-Bum, D. W. Moon, M. G. Proietti, J. M. Llorens, N. Garro, A. Cros, and A. García-Cristóbal, J. Microsc. (Paris) (to be published).

${ }^{15}$ J. Stangl, V. Holy, and G. Bauer, Rev. Mod. Phys. 76, 725 (2004).
${ }^{16}$ J.-L. Hodeau, V. Favre-Nicolin, S. Bos, H. Renevier, E. Lorenzo, and J.-F. Bérar, Chem. Rev. (Washington, D.C.) 101, 1843 (2001).

${ }^{17}$ W. A. Hendrickson, Science 254, 51 (1991).

${ }^{18}$ H. Stragier, J. O. Cross, J. J. Rehr, Larry B. Sorensen, C. E. Bouldin, and J. C. Woicik, Phys. Rev. Lett. 69, 3064 (1992); S. Grenier, M. G. Proietti, H. Renevier, L. Gonzales, J. M. Garcia, and J. Garcia, Europhys. Lett. 57, 499 (2002).

${ }^{19}$ A. Letoublon, V. Favre-Nicolin, H. Renevier, M. G. Proietti, C. Monat, M. Gendry, O. Marty, and C. Priester, Phys. Rev. Lett. 92, 186101 (2004).

${ }^{20}$ T. Shibata, K. Asai, S. Sumiya, M. Mouri, M. Tanaka, O. Oda, H. Katsukawa, H. Miyake, and K. Hiramatsu, Phys. Status Solidi C 0(7), 2023 (2003).

${ }^{21}$ U. Pietsch, V. Holy, and T. Baumbach, High-resolution X-ray Scattering: From thin films to lateral nanostructures (SpringerVerlag, Berlin, 2004).

${ }^{22}$ M. Schmidbauer, D. Grigoriev, M. Hanke, P. Schafer, T. Wiebach, and R. Kohler, Phys. Rev. B 71, 115324 (2005).

${ }^{23}$ V. Favre-Nicolin, Computer code, NANOMAD (private communication).

${ }^{24}$ M. G. Proietti, H. Renevier, J. L. Hodeau, J. Garcia, J. F. Berar, and P. Wolfers, Phys. Rev. B 59, 5479 (1999).

${ }^{25}$ H. Renevier, S. Grenier, S. Arnaud, J. F. Bérar, B. Caillot, J. L. Hodeau, A. Létoublon, M. G. Proietti, and B. Ravel, J. Synchrotron Radiat. 10, 435 (2003).

${ }^{26}$ A. L. Ankudinov, B. Ravel, J. J. Rehr, and S. D. Conradson, Phys. Rev. B 58, 7565 (1998).

${ }^{27}$ M. Newville, B. Ravel, D. Haskel, J. J. Rehr, E. A. Stern, and Y. Yacobi, Physica B 208-209, 154 (1995).

${ }^{28}$ F. Romanato, D. De Salvador, M. Berti, A. Drigo, M. Natali, M. Tormen, G. Rossetto, S. Pascarelli, F. Boscherini, C. Lamberti, and S. Mobilio, Phys. Rev. B 57, 14619 (1998). 
${ }^{29}$ H. Schulz and K. H. Thiemann, Solid State Commun. 23, 815 (1997)

${ }^{30}$ A. D. Andreev and E. P. O'Reilly, Phys. Rev. B 62, 15851 (2000).

${ }^{31}$ F. d'Acapito, F. Boscherini, S. Mobilio, A. Rizzi, and R. Lantier, Phys. Rev. B 66, 205411 (2002).
${ }^{32}$ M. Arlery, J. L. Rouviére, F. Widmann, B. Daudin, G. Feuillet, and H. Mariette, Appl. Phys. Lett. 74, 3287 (1999).

${ }^{33}$ N. Gogneau, E. Monroy, D. Jalabert, E. Sarigiannadou, J.-L. Rouviére, and B. Daudin, J. Appl. Phys. 96, 1104 (2004).

${ }^{34}$ A. Bourret, C. Adelmann, B. Daudin, J.-L. Rouviere, G. Feuillet, and G. Mula, Phys. Rev. B 63, 245307 (2001). 